\title{
EVIDÊNCIAS E DEPENDÊNCIA ONTOLÓGICA: SOBRE INTERPRETAÇÕES DE LÓGICAS PARACONSISTENTES ${ }^{1}$
}

\author{
Abílio Rodrigues ${ }^{2}$
}

Henrique Antunes ${ }^{3}$

\begin{abstract}
RESUMO
Neste texto, apresentamos duas abordagens distintas, mas não necessariamente excludentes, da paraconsistência. A primeira trata das lógicas da evidência e verdade (logics of evidence and truth), nas quais contradições são interpretadas como evidência não conclusiva, simultânea e conflitante para um par de proposições $A$ e $\neg A$. A segunda abordagem propõe que as diferenças entre a lógica clássica e alguns sistemas de lógica paraconsistente correspondem à distinção entre objetos ontologicamente independentes e ontologicamente dependentes, sendo esses últimos caracterizados como objetos cuja existência depende de nossos processos psicológicos e práticas linguísticas. Em ambas as alternativas, ilustramos como justificar a legitimidade das lógicas paraconsistentes sem endossar a tese de que a realidade, para ser descrita corretamente, requer proposições contraditórias.
\end{abstract}

Palavras-chave: Evidência. Dependência Ontológica. Contradição. Paraconsistência. Paracompletude.

\begin{abstract}
In this paper we present two distinct, though not necessarily incompatible, approaches to paraconsistency. The first approach concerns the logics of evidence and truth, wherein contradictions are interpreted as conflicting nonconclusive evidence for a pair of propositions $A \& \neg A$. The second approach proposes that the differences between classical logic and some systems of paraconsistent logic correspond to the distinction between ontologically independent and ontologically dependent objects, where the latter are characterized as objects whose existence depends on our psychological processes and linguistic practices. In either interpretation, we illustrate how to justify the

\footnotetext{
${ }^{1} \mathrm{O}$ primeiro autor agradece o apoio do $C N P q$ (Conselho Nacional de Desenvolvimento Científico e Tecnológico), projeto de pesquisa 311911/2018-8. Partes deste texto já foram publicadas em outros trabalhos dos autores, especialmente (Antunes 2019a) e (Carnielli e Rodrigues 2020).

2 Professor associado do Departamento de Filosofia da Universidade Federal de Minas Gerais. E-mail: abilio.rodrigues@gmail.com. ORCID: 0000-0001-6639-9550.

${ }^{3}$ Pesquisador de Pós-Doutorado do Departamento de Filosofia da Universidade de Minas Gerais. E-mail: antunes.henrique@outlook.com. ORCID: 0000-0003-2089-8957.
} 
legitimacy of paraconsistent logics without endorsing the thesis that reality itself requires contradictory propositions to be correctly described.

Keywords: Evidence. Ontological Dependence. Contradiction. Paraconsistency. Paracompleteness.

\section{Introdução}

Uma questão central na filosofia da lógica é se os princípios lógicos têm caráter ontológico, epistêmico ou linguístico, ou em outras palavras, como colocado por Popper (1963, pp. 206ss), se tais princípios dizem respeito à realidade, ao pensamento ou à linguagem. Esses três aspectos da lógica já podem ser implicitamente encontrados em Aristóteles, que formula na Metafísica três versões do princípio da não contradição, e cada uma delas pode ser relacionada, em algum sentido, com cada um desses aspectos ${ }^{4}$.

Chateaubriand (2001) defende que o caráter fundamental da lógica não é linguístico, mas sim metafísico, o que significa que a lógica tem, por um lado, um aspecto ontológico, como uma "teoria sobre as características mais gerais e universais da realidade," e por outro lado um aspecto epistêmico, como "parte de uma teoria geral do conhecimento" (Chateaubriand 2001, pp. 16). Tugendhat e Wolf (1996, cap. 1) apresentam o problema a partir de uma perspectiva histórica, relacionando esses três aspectos a diferentes períodos da história da filosofia, respectivamente antiga e medieval, moderna e contemporânea. De fato, um traço característico da filosofia analítica é ter colocado a análise da linguagem como uma ferramenta fundamental para a discussão filosófica, mas isso não significa que os aspectos ontológicos e epistêmicos da lógica tenham sido esquecidos pela filosofia contemporânea. Esses três aspectos, na verdade, não são mutuamente exclusivos e podem ser combinados. A lógica pode ser aplicada para representar diferentes contextos argumentativos e dependendo das características de tais contextos um caráter ontológico, epistêmico ou linguístico pode ser predominante.

\footnotetext{
${ }^{4}$ Essa leitura de Aristóteles é encontrada, por exemplo, em Gottlieb (2013seção 1), Łukasiewicz (1910, 509ss), e discutida em Carnielli e Rodrigues (2016seção 3.2.4). As três versões do princípio da não contradição estão na Metafísica de Aristóteles, respectivamente, 1005b15, 1005b25, 1011b15.
} 


\section{Consequência clássica}

No século XX, a lógica como campo de estudo foi dividida em diferentes disciplinas, mas é correto afirmar que o principal conceito da lógica é o de consequência lógica, de modo que a questão central da lógica é: $o$ que se segue de quê?

O tratamento da consequência que chamamos de lógica clássica foi inaugurado por (Frege 1879) ao apresentar o sistema formal da Begriffschrift, que tem como fragmento um sistema dedutivo da lógica clássica de primeira ordem. O sistema de Frege é apresentado sintaticamente, mas a ideia subjacente é que as regras de inferência preservam verdade. Essa ideia é expressa de modo preciso pela definição semântica de consequência clássica:

(1) $A$ segue de $\Gamma(\Gamma \vDash A)$ se e somente se para todo modelo $M$, se as premissas em $\Gamma$ são verdadeiras em $M$, então a conclusão $A$ é verdadeira em $M$,

onde um modelo para uma linguagem $L$ é um domínio $D$ e uma função $I$ que interpreta $L$ sobre $D$. A definição (1) expressa a ideia pré-teórica de consequência lógica segundo a qual um argumento é válido quando é impossível que suas premissas sejam verdadeiras e sua conclusão falsa.

Entre os princípios lógicos que são válidos na lógica clássica estão a não contradição, o terceiro excluído e o princípio da explosão, expressos em uma linguagem proposicional respectivamente pelas fórmulas

(2) $\vdash \neg(A \wedge \neg A)$,

(3) $\vdash A \vee \neg A$,

(4) $A, \neg A \vdash B$.

Nas leituras clássicas, o princípio da não contradição (2) afirma que uma contradição, i.e. a conjunção de uma proposição $A$ e sua negação $\neg A$, é sempre falsa, e o terceiro excluído (3) diz que dadas uma proposição $A$ e sua negação $\neg A$, pelo menos uma delas é verdadeira. O princípio da explosão (4), também 
chamado ex contradictione quodlibet, diz que qualquer proposição se segue de uma contradição.

A validade da explosão na lógica clássica é o resultado de considerar preservação de verdade não apenas como uma condição necessária, mas também como uma condição suficiente para consequência lógica. Como não há modelo $M$ tal que $A$ e $\neg A$ sejam verdadeiras em $M$, segue-se que para qualquer $B$, não há modelo $M$ tal que $A$ e $\neg A$ são verdadeiras e $B$ é falsa em $M$. O papel do princípio da explosão na lógica clássica é normativo, pois obviamente ele nunca é de fato aplicado. Enquanto (2) diz que uma contradição é sempre falsa, (4), por outro lado, diz algo mais forte: afirma as consequências inaceitáveis que se deveriam enfrentar se uma contradição fosse tomada como verdadeira. É mais eficaz, portanto, que a não contradição para proibir contradições.

Como resultado da definição de consequência clássica como preservação da verdade, e considerando que verdade depende da realidade, é correto atribuir à lógica clássica um caráter predominantemente ontológico, como também realista, dado que o terceiro excluído é válido. Isso não significa que a lógica clássica não possa ser interpretada sem ingredientes ontológicos. Um sistema formal não é comprometido com uma única interpretação, ainda que tenha sido proposto com uma determinada interpretação pretendida. Mas a lógica clássica se encaixa perfeitamente bem com uma concepção de verdade realista e que rejeita contradições verdadeiras, e também com uma concepção ontológica da lógica. As lógicas não clássicas que serão discutidas a seguir permitem combinar a consequência clássica em termos de preservação da verdade com diferentes tratamentos da consequência lógica, recuperando a lógica clássica de tal modo que essas características da negação clássica são preservadas

\section{Contextos contraditórios}

Embora a lógica clássica não tolere contradições, é um fato que elas aparecem em vários contextos argumentativos. Bancos de dados muitas vezes contêm não apenas informações incompletas, mas também contraditórias. In- 
vestigações científicas fornecem vários exemplos de situações reais em que contradições parecem ser inevitáveis ${ }^{5}$. Teorias matemáticas importantes permitiram provar resultados contraditórios no passado e, em virtude dos resultados de teoremas de incompletude de Gödel, não é possível saber com certeza se as teorias com as quais os matemáticos lidam hoje em dia estão livres de contradição. Personagens de ficção são algumas vezes descritas por seus autores como tendo propriedades contraditórias, seja intencional ou inadvertidamente. Ao contrário da lógica clássica, lógicas paraconsistentes são capazes de lidar com teorias e contextos argumentativos contraditórios.

Uma lógica é paraconsistente se, e somente se, tiver uma negação não explosiva, o que significa que o princípio da explosão (4) não é válido. Dada uma linguagem $L$ e uma relação de consequência $\vdash$, um conjunto $\Gamma$ de proposições de $L$ é chamado trivial se, para todas as proposições $A$ de $L, \Gamma \vdash A$. Um conjunto $\Gamma$ é contraditório se, para alguma proposição $A$ de $L, \Gamma \vdash A$ e $\Gamma \vdash \neg A$. Se o princípio da explosão for válido em $\vdash$, um conjunto $\Gamma$ contraditório será também trivial. Mas se a relação de consequência $\vdash$ for paraconsistente, $\vdash$ permite conjuntos de proposições contraditórios mas não triviais.

Uma vez que existem contextos contraditórios e não triviais, a questão que se coloca é pela natureza das contradições que são admitidas em tais contextos, a qual está diretamente relacionada aos três aspectos da lógica mencionados anteriormente. $\mathrm{O}$ dialeteísmo é a tese segundo a qual existem dialetéias, que são sentenças ou proposições $A$ tais que tanto $A$ quanto sua negação $\neg A$ são verdadeiras (cf. Priest 1998, 2006; Priest, Berto, e Zach 2018). Se se admite que é a realidade que torna verdadeira uma proposição verdadeira, o dialeteísmo fornece uma resposta ontológica à pergunta pela natureza das contradições porque fica comprometido com o que chamamos de 'contradi-

\footnotetext{
${ }^{5}$ Existem teorias científicas bem-sucedidas em suas áreas de conhecimento que produzem contradições em determinadas circunstâncias específicas, ou quando combinadas com outras teorias também bem-sucedidas. Um exemplo, discutido em Carnielli e Rodrigues (2020seção 3.2.1), diz respeito ao surgimento da teoria da relatividade, que resolveu uma contradi ção entre a mecânica clássica e a teoria do campo eletromagnético. Sobre inconsistências em teorias científicas, ver e.g. (Colyvan 2008; da Costa e French 2003; da Costa e Krause 2014; Meheus 2002).
} 
ções reais,' i.e., aspectos da realidade que para serem descritos corretamente exigem proposições contraditórias. ${ }^{6}$

Porém, essa não é a única maneira de justificar a rejeição do princípio da explosão pelas lógicas paraconsistentes. Contradições podem ser relacionadas a um conceito epistêmico, como justificativas não conclusivas e conflitantes, ou ter origem no pensamento ou no uso da linguagem. Em ambos os casos, proposições $A$ e $\neg A$ que ocorrem em contextos contraditórios não são verdadeiras no sentido forte de serem tornadas verdadeiras por algum aspecto da realidade que seja intrinsecamente contraditório.

O objetivo deste texto é apresentar duas abordagens à paraconsistência que não estão comprometidas com contradições reais. A primeira, discutida na seção 2, trata das lógicas da evidência e verdade (logics of evidence and truth, (Carnielli e Rodrigues 2017; Rodrigues, Bueno-Soler, e Carnielli 2020)), nas quais contradições são interpretadas como evidência não conclusiva, simultânea e conflitante para $A$ e $\neg A$. Essa abordagem é denominada 'epistêmica' porque rejeita contradições verdadeiras e enfatiza o ingrediente epistêmico presente na noção de evidência. A segunda abordagem, que é o foco da seção 3, propõe que as diferenças entre a lógica clássica e alguns sistemas de lógica paraconsistente correspondem à distinção entre objetos ontologicamente independentes e ontologicamente dependentes, sendo estes últimos caracterizados como objetos que dependem de nossos processos psicológicos e práticas linguísticas (cfe. Azzouni 2004). De acordo com essa abordagem, não há contradições acerca da realidade extra-linguística e extramental, muito embora contradições possam ser produzidas pela linguagem e

${ }^{6}$ Priest $(2019,588-89)$ afirma que a concepção de dialeteísmo por ele endossada não é comprometida com contradições na realidade, mas admite que essa leitura do dialeteísmo não é incomum. O que nós diríamos é que essa leitura do dialeteísmo de Priest é na verdade a predominante - ver, a título de exemplo, Batens $(2002,138)$, N. M. Kiefer $(2019,232)$, da Costa e O. Bueno (2007, 900), da Costa e French (2003, 106), da Costa e Krause (2014, 3041), Frápolli (1996, 438), Garfield (2004, 237), Mares (2004, 265), Meheus (2002, viii), H. Wansing $(2016,180)$, Steinberger (2016, footnote 2), entre outros. Além disso, também diferentemente do que lemos em (Priest 2019), pelo menos prima facie há suporte textual para uma leitura ontológica do dialeteísmo. Em Priest, Berto, e Zach (2018, Sect. 3.4), lemos que algumas dialetéias envolvem "contradições que afetam objetos concretos e o mundo empírico," e em Priest $(2006,159)$ que "devemos agora analisar contradições que surgem em domínios mais concretos, e especialmente no mundo empírico.” Note-se, entretanto, que legitimidade de uma abordagem não ontológica da paraconsistência, que não considera verdadeiras as proposições contraditórias de contextos paraconsistentes, não depende dessa discussão acerca do dialeteísmo de Priest. 
pelo pensamento - as quais dizem respeito ao que chamamos, seguindo (Azzouni 2004), de objetos ontologicamente dependentes.

\section{Uma abordagem epistêmica}

\section{Contradições verdadeiras?}

Um contra-exemplo ao princípio da explosão consiste em uma circunstância na qual um par de proposições $A$ e $\neg A$ vale mas alguma proposição $B$ não vale. A proposta do dialeteísmo, como vimos, é que existem circunstâncias em que proposições $A$ e $\neg A$ são verdadeiras, muito embora o mundo não seja trivial (i.e., nem tudo o mais também seja verdadeiro), e isso seria suficiente como contraexemplo para o princípio da explosão. O dialeteísmo é sem dúvida uma visão não ortodoxa, discutida principalmente no contexto da filosofia da lógica. Matemáticos e investigadores das ciências empíricas não são muito interessados na questão de se existem ou não descrições da realidade contraditórias e verdadeiras, eles simplesmente pressupõem que não existem.

A formulação padrão do princípio da não contradição em termos de objetos e propriedades, que corresponde à versão ontológica da não contradição em Aristóteles [1005b15] e é amplamente aceita, é a seguinte:

(5) Não é possível um objeto $a$ possuir e não possuir uma propriedade $P$ ao mesmo tempo e no que diz respeito ao mesmo aspecto.

Uma contradição verdadeira portanto dependeria de um objeto $a$ que possui não possui simultaneamente uma propriedade $P$ ao mesmo tempo e no que diz respeito ao mesmo aspecto. Essas duas condições eliminam supostas contradições que dependem de perspectivas diferentes, como por exemplo estar frio e quente (i.e. não estar frio) para duas pessoas acostumadas com climas muito quentes ou muito frios, e também propriedades instanciadas em diferentes momentos, como por exemplo ser jovem e não ser jovem ${ }^{7}$.

\footnotetext{
${ }^{7} \mathrm{Na}$ tradição filosófica ocidental, os pensadores frequentemente mencionados como tendo defendido que a realidade é (em algum sentido) contraditória são Heráclito e Hegel. Mas em ambos as contradições estão relacionadas ao movimento e à mudança, e portanto é perfeita-
} 
O apelo a propriedades pode ser dispensado. Basta recorrer à relação de satisfação utilizada por (Tarski 1956) para definir verdade, e uma contradição verdadeira $P a \wedge \neg P a$ dependeria então de um objeto $a$ que satisfaz simultaneamente predicados $P$ e $\neg P$. Assim, não é necessário apelar a entidades como fatos ou estados de coisas contraditórios, mas apenas a objetos contraditórios. De todo modo, a existência de dialetéias depende, na melhor hipótese, da existência de objetos contraditórios. Por essa razão, e em consonância com a leitura padrão do dialeteísmo, dizemos que o dialeteísmo fornece uma motivação ontológica para rejeição do princípio da explosão.

\section{Evidência e verdade}

O princípio da explosão pode ser rejeitado por razões puramente epistêmicas. A paraconsistência pode ser combinada com uma visão realista e não dialeteísta da verdade que simultaneamente aceita o terceiro excluído e rejeita contradições verdadeiras ${ }^{8}$. Segundo esse ponto de vista, as contradições que ocorrem em diversos contextos argumentativos não significam que proposições $A$ e $\neg A$ sejam verdadeiras, mas apenas que existem evidências conflitantes para $A$ e $\neg A$, e evidência para uma proposição $A$ não implica a verdade de $A$ (voltamos a esse ponto em breve).

Circunstâncias desse tipo ocorrem, por exemplo, nas ciências empíricas. Faz parte da rotina de físicos lidar com teorias que produzem contradições em circunstâncias críticas ou quando colocadas junto com outras teorias. O fato que quase todas as teorias científicas em algum ponto de seu desenvolvimento são contraditórias ou incompatíveis com outras teorias não é um problema para a racionalidade (cfe. Meheus 2002, vii). Há contradições que a prática científica não pode deixar de lidar, e contextos contraditórios temporá-

mente possível interpretá-los de tal modo que (5) não seja violado. A interpretação segundo a qual não há, nos fragmentos de Heráclito, contradições mas sim oposições e conflitos entre forças, estados e perspectivas é bem estabelecida na literatura. Quanto a Hegel, entretanto, dado que há trechos que mencionam explicitamente o princípio da não contradição, não fica claro à primeira vista que as 'contradições hegelianas' não são de fato violações do princípio da não contradição tal como formulado em (5) acima. Para um panorama sobre como contradições foram tratadas na história da filosofia, incluindo Heráclito e Hegel, ver (Rodrigues 2017).

${ }^{8}$ Sobre como conciliar uma visão realista da lógica com lógicas não clássicas, inclusive a paraconsistente, ver (Frade e Rodrigues 2020). 
rios são um ingrediente essencial da prática científica (cfe. Nickles 2002, 2). Contradições podem ter origem em limitações do nosso aparato cognitivo, falhas em instrumentos de medida, estágios no desenvolvimento das teorias, ou simplesmente erros que podem ou não ser percebidos e corrigidos. Em todos esses casos, contradições são relacionadas em primeiro lugar ao pensamento e ao processo de aquisição de conhecimento, e tais contradições são chamadas de epistêmicas (cf. Carnielli e Rodrigues 2020, seção 3.2).

Considere um contexto argumentivo, como os mencionados acima, em que um par de proposições $A$ e $\neg A$ são admitidas. A questão, então, é a seguinte: qual é a propriedade que vamos atribuir a $A$ e $\neg A$ tal que seria possível uma proposição ter essa propriedade mesmo sem ser verdadeira? Tal propriedade tem de ser mais fraca que verdade, no sentido que uma proposição poderia ter essa propriedade sem ser verdadeira.

Evidência para uma proposição $A$ é explicada informalmente em (Carnielli e Rodrigues 2017) como 'razões para acreditar em A.' Tais razões são objetivas, podem não ser conclusivas (evidência para $A$ não implica a verdade de $A$ ), e não requerem crença por parte de um agente (evidência para $A$ não implica que algum agente acredita em $A$ ). Evidência pode também ser explicada como um conteúdo semântico mais uma justificativa, que pode não ser conclusiva ou mesmo errada (cf. Carnielli e Rodrigues 2020; Rodrigues, Bueno-Soler, e Carnielli 2020). O ingrediente epistêmico da noção de evidência reside nessa justificativa que pode, no fim das contas, não ser uma justificativa efetiva. A noção de evidência, portanto, é uma propriedade mais fraca que verdade e fornece uma explicação intuitiva para a paraconsistência sem recorrer à contradições verdadeiras.

\section{Informações contraditórias}

Outra abordagem não dialeteísta da paraconsistência, no sentido de admitir contradições sem considerá-las verdadeiras, foi proposta nos anos 1970 por Belnap (1977b, 1977a) e Dunn (1976). A motivação de Belnap ao propor uma semântica de quatro valores para a lógica FDE (logic of firstdegree entailment) era fornecer uma lógica a ser usada por um computador 
que recebe informações não confiáveis de diferentes fontes. Essas informações podem ser incompletas ou contraditórias e são possíveis quatro cenários, representados pelos valores semânticos Verdadeiro, Falso, Nenhum e Ambos. Os valores Verdadeiro e Falso não são pensados como a verdade e a falsidade clássicas. Eles são explicados em termos de um computador que recebe a informação ('a computer being told') de que uma proposição é verdadeira ou falsa (Belnap 1977b, pp. 34-35). Esses quatro valores significam, respectivamente, que o computador recebe apenas a informação de que uma proposição $A$ é verdadeira ('just told true'), apenas a informação de que $A$ é falsa ('just told false'), não recebe nehuma informação sobre $A$ ('told neither true nor false'), e recebe a informação de que $A$ é verdadeira e a informação de que ela também é falsa ('told both true and false'). Belnap (1977b, pp. 47) afirma explicitamente que as proposições são verdadeiras ou falsas independentemente da informação que foi inserida no computador. Um computador pode receber a informação de que $A$ é verdadeira (resp. falsa), mesmo que $A$ seja falsa (resp. verdadeira). Assim como evidência, a noção de um computador receber a informação ('being told') de que uma proposição é verdadeira ou falsa é mais fraca que a noção de verdade, pois o computador pode receber a informação de que $A$ é verdadeira mesmo que $A$ seja falsa.

\section{Lógicas da evidência e verdade}

\section{O operador de classicalidade}

da Costa $(1963,1974)$, na sua hierarquia de lógicas $C_{n}$, propôs um 'operador de bom comportamento,' definido em $C_{1}$ em termos de não contradição,

(6) $A^{\circ}:=\neg(A \wedge \neg A)$

que divide as proposições de uma dada linguagem em dois grupos: aquelas para as quais contradições são toleradas e as que contradições não são toleradas. 
Pela definição (6) acima, uma proposição $A$ é 'bem comportada,' expresso por $A^{\circ}$, quando o princípio da não contradição vale para $A$.

As LFIs (logics of formal inconsistency) são um desenvolvimento da proposta de da Costa em que um operador de consistência o é concebido de tal modo que $\circ A$ pode não ser equivalente a $\neg(A \wedge \neg A)$ (Carnielli e Coniglio 2016; Carnielli, Coniglio, e João Marcos 2007; Carnielli, Coniglio, e Rodrigues 2019). Nas LFIs, 。 recupera o princípio de explosão para proposições no seu escopo:

(7) $A, \neg A \nvdash B$, ao passo que $\circ A, A, \neg A \vdash B$.

Embora na maior parte das LFIs investigadas na literatura a função do operador $\circ$ esteja circunscrita a recuperar o princípio de explosão (e inferências que dele se seguem), ele também pode desempenhar um papel mais geral em lógicas que, além da explosão, também invalidam o terceiro excluído. Em outras palavras, além de satisfazer (7), ○ também permite recuperar $A \vee \neg A$ :

(8) $\forall A \vee \neg A$, ao passo que $\circ A \vdash A \vee \neg A$.

As LFIUs (logics of formal inconsistency and undeterminedness) são lógicas nas quais $\circ$ tem ambas as propriedades (7) e (8), e nesse caso ele costuma ser chamado operador de classicalidade (e.g. Carnielli e Rodrigues 2017; Omori e Sano 2014; Rodrigues, Bueno-Soler, e Carnielli 2020; Szmuc 2016)9 .

\section{As lógicas BLE e $L E T_{J}$}

Em (Carnielli e Rodrigues 2017), duas lógicas paraconsistentes são propostas: BLE (basic logic of evidence), que é a lógica $N 4$ interpretada

\footnotetext{
${ }^{9}$ Tal como ocorre no caso das LFIs que validam o terceiro excluído irrestritamente, lógicas que são paracompletas (i.e., nas quais o terceiro excluído não é válido), mas não paraconsistentes também podem incluir em sua linguagem um operador ○ que satifaça apenas (8). Nesse caso, a lógica resultante é chamada logic of formal undeterminedness $(L F U)$. Note que embora nem toda $L F I$ ou $L F U$ seja também uma $L F I U$, toda $L F I U$ é tanto uma $L F I$ quanto uma $L F I U$.
} 
em termos de preservação de evidência ${ }^{10}$, e $L E T_{J}$ (logic of evidence and truth based on intuitionistic positive logic). $L E T_{J}$ é uma $L F I U$ que estende BLE e na qual o operador $\circ$ permite lidar simultaneamente com contextos de dois tipos, um sujeito à lógica clássica, em as inferências preservam verdade, e outro sujeito a $B L E$ em que as inferências preservam evidência.

A lógica $B L E$ é definida em uma linguagem proposicional com os conectivos $\neg, \wedge, \vee \mathrm{e} \rightarrow$ pelas seguintes regras de dedução natural:

$$
\frac{A \quad B}{A \wedge B} \wedge I \quad \frac{A \wedge B}{A} \wedge E \frac{A \wedge B}{B}
$$

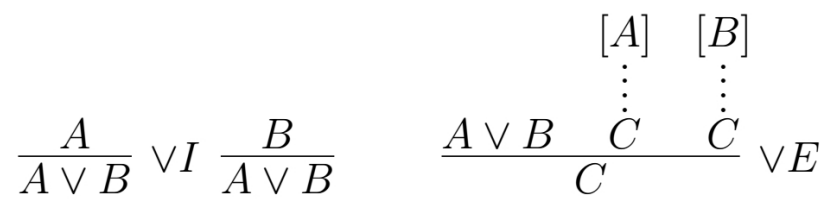

$$
\begin{aligned}
& {[A]} \\
& \frac{\dot{B}}{A \rightarrow B} \rightarrow I \quad \frac{A \rightarrow B \quad A}{B} \rightarrow E
\end{aligned}
$$

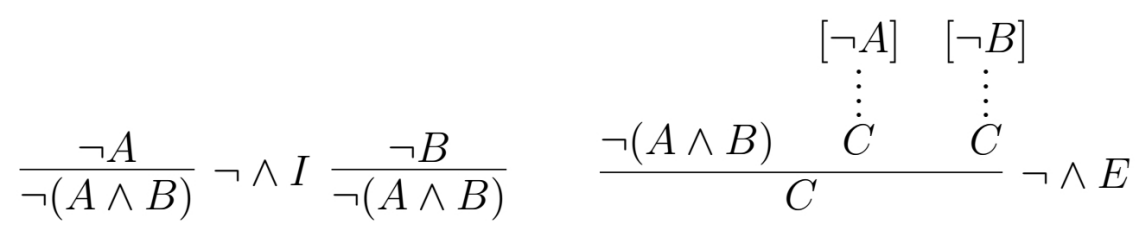

$$
\begin{aligned}
& \frac{\neg A \quad \neg B}{\neg(A \vee B)} \neg \vee I \quad \frac{\neg(A \vee B)}{\neg A} \neg \vee E \frac{\neg(A \vee B)}{\neg B} \\
& \frac{A \neg \neg}{\neg(A \rightarrow B)} \neg \rightarrow I \quad \frac{\neg(A \rightarrow B)}{A} \neg \rightarrow E \frac{\neg(A \rightarrow B)}{\neg B} \\
& \frac{A}{\neg \neg A} D N \frac{\neg \neg A}{A}
\end{aligned}
$$

As regras de $B L E$ podem ser divididas em três grupos: (a) regras de introdução e eliminação de $\wedge, \vee$ e $\rightarrow$; (b) regras de introdução e eliminação de $\wedge, \vee$ e $\rightarrow$ negadas; (c) regras de introdução e eliminação da dupla negação.

${ }^{10}$ Sobre BLE e N4, ver seção 5.3 e nota de rodapé 21 de (Carnielli e Rodrigues 2017). 
A dupla negação não é suficiente para obter a lógica clássica porque em $B L E$ uma fórmula $\neg \neg A$ somente pode ser introduzida a partir de uma premissa $A$. Isso ocorre porque não há regra de introdução da negação em $B L E$, i.e. a inferência abaixo não é válida:

(9) $A \rightarrow B, A \rightarrow \neg B \vdash \neg A$.

A implicação de $B L E$ é não relevante, o que significa que vale o princípio conhecido como ex quodlibet verum, que corresponde à fórmula

$$
(10) \vdash A \rightarrow(B \rightarrow A) \text {. }
$$

O que (10) diz é que sendo $A$ o caso, qualquer proposição implica $A$. Portanto, um contraexemplo para (9) é dado por uma circunstância em que há evidência conflitante para $B$ e $\neg B$ mas nenhuma evidência para $\neg A$. A equivalência entre $A$ e $\neg \neg A$ diz que 'evidência de que é falso que $A$ é falso' é o mesmo que 'evidência de que $A$ é verdadeiro.'

Os grupos (a) e (b) expressam, respectivamente, evidência positiva e negativa para conjunção, disjunção e implicação. Regras positivas e negativas independentes são necessárias porque evidência positiva e negativa são independentes e não complementares. Por essa razão, os quatro cenários abaixo são possíveis:

i. Apenas evidência que $A$ é verdadeira: $A$ vale, $\neg A$ não vale;

ii. Apenas evidência que $A$ é falsa: $\neg A$ vale, $A$ não vale;

iii. Ausência de evidência: nem $A$ nem $\neg A$ vale;

iv. Evidência conflitante: ambas $A$ e $\neg A$ valem.

A lógica $L E T_{J}$ é definida em uma linguagem que estende a linguagem de $B L E$ com o operador de classicalidade $\circ$ e pelo acréscimo das seguintes regras ao sistema de dedução natural de $B L E$ : 


$$
\frac{\circ A \quad A \quad \neg A}{B} E X P^{\circ} \quad \frac{\circ A}{A \vee \neg A} P E M^{\circ}
$$

As regras $E X P^{\circ}$ e $P E M^{\circ}$ recuperam a lógica clássica para as proposições no escopo de $\circ$, ou formadas com tais proposições e os conectivos $\neg, \wedge, \vee, \mathrm{e} \rightarrow(\mathrm{Car}$ nielli e Rodrigues 2017, Fact 18). A lógica $L E T_{J}$ é capaz de expressar os seguintes seis cenários:

- Quando $\circ A$ não vale, i.e. não há evidência conclusiva, temos os quatro cenários i-iv acima.

- Quando $\circ A$ vale, i.e. há evidência conclusiva, dois cenários são acrescentados:

v. $A$ vale: evidência conclusiva para $A$, i.e. $A$ é verdadeira; vi. $\neg A$ vale: evidência conclusiva para $\neg A$, i.e. $A$ é falsa.

$L E T_{J}$ trata evidência conclusiva como verdade porque considera que evidência conclusiva é sujeita à lógica clássica. $L E T_{J}$ pode também ser interpretada em termos de informação positiva ou negativa, que pode ser confiável ou não confiável. Nesse caso, os cenários i-iv são interpretados como informação não confiável e correspondem aos quatro valores da lógica $F D E$ que vimos na seção 2.3, e os cenários v e vi são interpretados como informação confiável, e esta é considerada sujeita à lógica clássica (cfe. Antunes et al. 2020).

A lógica $L E T_{J}$ expressa em um mesmo sistema formal duas noções de consequência, uma que preserva verdade (proposições sujeitas à lógica clássica) e outra que preserva evidência. O operador o pode ser considerado um comutador de contexto que muda o contexto de determinadas proposições, sujeitas à lógica clássica. $L E T_{J}$, portanto, é capaz de expressar contextos multilógicos e é alinhada a uma concepção pluralista da lógica.

Segundo a interpretação pretendida, $L E T_{J}$ expressa uma abordagem da paraconsistência diametralmente oposta ao dialeteísmo. Se uma proposição $A$ é verdadeira, i.e. se há evidência conclusiva para $A$, isso é expressado em $L E T_{J}$ pela conjunção $A \wedge \circ A$, e a falsidade de $A$, i.e. a verdade de $\neg A$, é ex- 
pressada por $\neg A \wedge \circ A$. Assim, se $A$ e $\neg A$ forem simultaneamente verdadeiras, temos $A \wedge \neg A \wedge \circ A$, e trivialidade se segue pela regra $E X P^{\circ}$. De acordo com a interpretação pretendida de $L E T_{J}$, portanto, contradições verdadeiras não são toleradas porque resultam em trivialidade, assim como na lógica clássica.

\section{Uma abordagem nominalista}

Como acabamos de ver, a abordagem epistêmica da paraconsistência fornece motivações bastante sólidas para rejeitar o princípio da explosão sem que seja necessário endossar a tese dialeteísta de que existem contradições verdadeiras. Vimos também que de acordo com essa interpretação não há um conflito real entre a lógica paraconsistente e a lógica clássica, uma vez enquanto a primeira fornece um tratamento da consequência lógica como preservação de verdade, a segunda trata da preservação de evidência (ou informação), que é uma noção reconhecidamente mais fraca.

Nesta seção, apresentaremos uma abordagem alternativa à paraconsistência segundo a qual as diferenças entre a lógicas clássica e alguns sistemas de lógica paraconsistente repousam em determinadas distinções ontológicas fundamentais entre dois tipos de objeto, entidades ontologicamente independentes e ontologicamente dependentes - uma distinção proposta por Jody (Azzouni 2004) no contexto do debate ontológico em filosofia da matemática ${ }^{11}$. A ideia central é que embora a lógica clássica forneça uma teoria satisfatória acerca de nosso raciocínio sobre domínios constituídos por objetos do primeiro tipo, determinados sistemas de lógica paraconsistente resultam ser mais adequados para raciocinar acerca de objetos do segundo.

Em virtude do fato de a distinção entre objetos ontologicamente dependentes e independentes ter sido proposta por Azzouni para sustentar a tese nominalista de que objetos matemáticos não existem, chamaremos essa segun-

\footnotetext{
11 As ideias a serem discutidas aqui foram originalmente apresentadas em (Antunes 2018, 2019a).
} 
da abordagem de nominalista, muito embora não trataremos dos problemas relacionados à ontologia da matemática neste artigo ${ }^{12}$.

Diferentemente da interpretação epistêmica da paraconsistência, a abordagem nominalista não afirma que contradições não possam ser verdadeiras em absoluto, mas apenas que elas não podem ser verdadeiras sobre a realidade extra-linguística e extra-mental. Nesse sentido, contradições verdadeiras são vistas como um mero subproduto da maneira como a linguagem e o pensamento operam e não possuem nenhum correspondente na realidade. Há que se notar, contudo, que as duas abordagens não são necessariamente incompatíveis, pois enquanto a abordagem epistêmica trata a noção de verdade em um sentido estritamente realista (i.e., verdade como correspondência), a abordagem nominalista considera também a possibilidade de enunciados serem verdadeiros ou falsos em virtude de determinadas práticas linguísticas ou estipulações. Como veremos agora, é exclusivamente com relação a verdades desse segundo tipo que a abordagem nominalista admite a possibilidade de algumas contradições serem verdadeiras.

\section{Objetos ontologicamente dependentes vs. independentes}

Grosso modo, objetos ontologicamente dependentes são aqueles cujas propriedades dependem inteiramente dos processos psicológicos ou das práticas linguísticas de um indivíduo ou grupo de indivíduos, ao passo que objetos ontologicamente independentes são entidades cujas propriedades não dependem de tais processos ou práticas, mas da maneira como a realidade extra-linguística e mental (i.e., o mundo) se configura. Como exemplos paradigmáticos de entidades do primeiro tipo, temos figuras sonhadas ou imaginadas, alucinações e personagens de ficção; e como exemplos paradigmáticos de entidades do segundo tipo, temos objetos macrofísicos, tais como pessoas, animais, planetas etc.

\footnotetext{
12 Ao invés de lidar com os objetos postulados pela matemática, como números, conjuntos, funções etc., consideramos apenas exemplos que envolvem personagens de ficção. A razão é que de acordo a proposta de Azzouni, tanto objetos matemáticos quanto ficcionais são ontologicamente dependentes, o que, segundo ele, significa que eles não existem realmente. Para uma discussão acerca das similaridades entre objetos matemáticos e ficcionais, ver (Azzouni 2015).
} 
Embora esses dois tipos de objeto sejam fundamentalmente distintos, asserimos ordinariamente enunciados que são aparentemente verdadeiros ou falsos acerca de ambos, sem que as sentenças asseridas revelem qualquer diferença entre eles, seja em sua forma ou através de algum vocábulo específico da linguagem natural. Dizemos, por exemplo, que Dom Quixote inspirou muitas outras personagens famosas assim como que Miguel de Cervantes inspirou muitos outros autores da literatura mundial; ou que Dr. Jekyll e Mr. Hyde são a mesma personagem assim como que Fernando Pessoa e Alberto Caeiro são a mesma pessoa.

A despeito de não dispormos de meios linguísticos distintivos que tornem explícito quando estamos falando de entidades ontologicamente dependentes ou independentes, é natural supor que não raciocinamos acerca de ambos os tipos de objeto exatamente da mesma maneira - ou, mais precisamente, que eles não estejam sujeitos às mesmas relações de consequência lógica. Com efeito, personagens de ficção não parecem atender a determinados princípios característicos da lógica clássica, como o terceiro excluído ou a explosão, uma vez que elas são frequentemente caracterizadas de maneira incompleta e, em alguns casos especiais, até mesmo contraditória. Por outro lado, entidades ontologicamente independentes representam os candidatos mais naturais de objetos sobre os quais raciocinamos classicamente.

A tese de que existem enunciados acerca de entidades ontologicamente dependentes que não satisfazem o terceiro excluído não apresenta maiores dificuldades. Afinal, obras literárias raramente fornecem uma descrição exaustivamente completa de todas as suas personagens. Por outro lado, a ideia de que entidades ontologicamente dependentes sejam suscetíveis a descrições contraditórias é um tanto menos plausível. No entanto, é fácil fornecer exemplos que mostram justamente isso, como a seguinte história apresentada por Everett (2005, pp. 633-34):

Quando chegou à terra da Dialetéia, Jane conheceu Jules e Jim. Isso a deixou confusa, uma vez que Jules e Jim eram e não eram pessoas distintas. No início, ela teve dificuldades para saber como deveria interagir com eles. Por exemplo, como Jules era e não era Jim, se Jim viesse à casa de Jane para tomar chá, Jules viria e não viria junto com ele. E nessas ocasiões ela não sabia quantos biscoitos servir. 
Jane se deu conta afinal o que fazer. Ela deveria comprar e não comprar mais biscoitos sempre que Jim viesse à sua casa. Depois disso, tudo ficou bem. (nossa tradução)

De acordo com essa história, Jim e Jules são e não são a mesma pessoa, o que implica que as personagens de Jim e Jules também são e não são a mesma ${ }^{13}$. Portanto, temos aqui um exemplo de objetos ontologicamente dependentes, viz., as personagens em questão, que satisfazem descrições contraditórias. E uma vez que o princípio da explosão proíbe toda e qualquer contradição, uma lógica adequada para raciocinar acerca de objetos desse tipo tem de ser paraconsistente.

Não pretendemos argumentar exaustivamente aqui a favor da tese que alguns objetos ontologicamente dependentes possuem propriedades contraditórias, ou que eles são suscetíveis a descrições contraditórias. No entanto, exemplos como o apresentado acima representam uma motivação inicial bastante forte a favor da tese de que enquanto a lógica clássica é correta para modelar nossos raciocínios acerca de objetos ontologicamente independentes, não se pode dizer o mesmo acerca de entidades ontologicamente dependentes. Afinal, em se tratando de objetos cujas propriedades foram todas estipuladas, não parece haver razões pelas quais algumas dessas estipulações não pudessem ser contraditórias, como no caso do fornecido por Everett. Assim, é possível distinguir entre as seguintes proposições:

(11) Não há objetos contraditórios; e

(12) Não há objetos ontologicamente independentes contraditórios,

onde (11) expressa que nenhum objeto, seja ele ontologicamente independente ou não, é passível de descrições contraditórias, ao passo que (12) restringe essa exigência apenas a objetos que existem independentemente de nós.

\section{A lógica LFI1}

\footnotetext{
${ }^{13}$ Parece ser indisputável que o único critério para determinar se duas personagens de uma mesma obra de ficção são ou não a mesma é se os indivíduos aos quais elas correspondem na história contada na obra são ou não idênticos. Afinal, que outra razão poderia existir para di zer que as personagens de Dr. Jekyll e de Mr. Hyde são a mesma, senão o fato de que esses indivíduos são, na verdade, a mesma pessoa de acordo com o livro de R. L. Stevenson?
} 
O exemplo acima sugere que enquanto devemos subscrever a (12), (11) é um princípio demasiadamente forte, i.e., que não há objetos contraditórios com exceção de alguns daqueles que são produzidos por nossas práticas linguísticas e processos psicológicos. Há que se perguntar agora como formular uma lógica que incorpore (12), ou seja, que proíba contradições sobre objetos que existem independentemente de nós, mas que ainda assim seja suficientemente liberal para permitir algumas contradições. Uma alternativa seria, naturalmente, adotar lógicas distintas a depender da natureza dos objetos em questão; i.e., adotar a lógica clássica para raciocinar acerca de objetos ontologicamente independentes e uma lógica paraconsistente e paracompleta para raciocinar acerca de objetos ontologicamente dependentes. Essa proposta padece, porém, de uma séria limitação, uma vez que frequentemente lidamos com argumentos que envolvem enunciados mistos, por exemplo:

- Dom Quixote é mais famoso que Mr. Hyde.

- Mr. Hyde é mais famoso que R. L. Stevenson.

- Logo, Dom Quixote é mais famoso que R. L. Stevenson.

Para lidar com argumentos desse tipo, nos quais algumas proposições dizem respeito tanto a objetos ontologicamente dependentes quanto independentes, precisamos, portanto, de uma única lógica que permita raciocinar acerca de ambos os tipos de objeto indistintamente.

As LFIs e LFIUs, apresentadas na seção anterior, oferecem as ferramentas necessárias para contornar essa dificuldade, pois a presença do operador de classicalidade, ॰, que permite distinguir contradições explosivas de não explosivas, possibilita expressar (12) perfeitamente bem, como veremos agora.

Considere a lógica (de primeira ordem) que resulta de acrescentarmos as seguintes regras a $B L E$ (cfe. seção 2.4.2): 


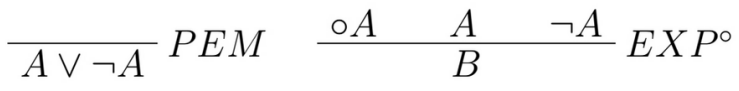

$$
\begin{aligned}
& \frac{\neg \circ A}{A} \neg \circ E \frac{\neg \circ A}{\neg A}
\end{aligned}
$$

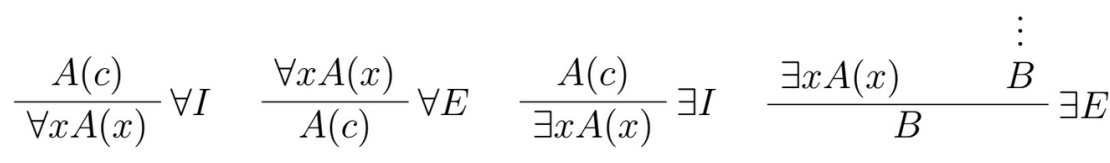

onde $A(c)$ é o resultado de se substituírem todas as ocorrências livres de $x$ em $A(x)$ pela constante individual $c$ e onde as regras para os quantificadores estão sujeitas às restrições usuais ${ }^{14}$.

Essa lógica é conhecida como $L F I 1$ e foi introduzida pela primeira vez em (Carnielli, João Marcos, e de Amo 2000) para modelar bancos de dados contraditórios (ver também (Amo, Carnielli, e João Marcos 2002; Omori e Waragai 2011)). Note que em virtude da presença da regra PEM, LFI1 não é uma $L F I U$, mas poderia ser facilmente modificada de modo que o sistema resultante fosse capaz de lidar com contextos nos quais o princípio do terceiro excluído não vale ${ }^{15}$. Além das regras de $B L E$ e das regras para os quantificadores, $L F I 1$ possui duas novas regras envolvendo $\circ$, que são as duas versões de $\neg$ $\circ$ E. Elas permitem provar que:

$$
(13) \vdash \neg \circ A \leftrightarrow(A \wedge \neg A),
$$

que expressa que sempre que se uma proposição não tem um comportamento clássico, ela é contraditória. Ao contrário do que se possa esperar, entretanto, que uma proposição $A$ não seja contraditória não implica $\circ A$ em $L F I 1$ :

$$
(14) \nvdash \neg(A \wedge \neg A) \rightarrow \circ A \text {. }
$$

\footnotetext{
${ }^{14} \mathrm{Em} \forall I$ e $\neg \exists I, A(c)(\neg A(c))$ não depende de nenhuma hipótese na qual $c$ ocorra; e em $\exists E$ e $\neg \forall E, c$ não ocorre em $B$ e em nenhuma hipótese da qual $A(c)(\neg A(c))$ dependa, com exceção possivelmente de $A(c)(\neg A(c))$.

15 Ver (Omori e Waragai 2011; Sano e Omori 2013; Omori e Sano 2014), onde a lógica $B S 4$, que é uma versão paracompleta de $L F I 1$, é investigada.
} 
A razão é que $\neg$ é uma negação paraconsistente e, assim, não proíbe que $A \wedge$ $\neg A$ e $\neg(A \wedge \neg A)$ valham simultaneamente ${ }^{16}$.

Uma propriedade muito importante de LFII é que se determinados predicados $P, Q, \ldots$ da linguagem não admitem instâncias contraditórias,

(15) $\forall x \circ P(x)$,

(16) $\forall x \forall y \circ Q(x, y)$,

então qualquer fórmula formada por meio de $P$ e $Q$, e.g.,

(17) $\forall x(P(x) \rightarrow \exists y(Q(x, y) \wedge \neg P(y)))$,

também não pode ser contraditória (sob pena de trivialidade). Ou seja, sempre que (15) e (16) valem, a seguinte fórmula também vale:

$(18) \circ(\forall x(P(x) \rightarrow \exists y(Q(x, y) \wedge \neg P(y))))$.

Isso significa que em LFII podemos identificar os contextos em que estamos justificados a raciocinar classicamente como aqueles nos quais todos os raciocínios envolvidos tratam de propriedades que não admitem contradições (o que, no exemplo acima, corresponde a assumir (15) e (16)), uma vez que qualquer contradição envolvendo essas propriedades levaria inevitavelmente à trivialidade, exatamente como na lógica clássica ${ }^{17}$.

\section{Independência ontológica e não contradição}

Voltemos à questão de como expressar (12) em LFI1. Uma alternativa seria argumentar que é possível identificar claramente as propriedades que apenas objetos ontologicamente independentes têm e exigir que todos os pre-

\footnotetext{
${ }^{16} \mathrm{Na}$ verdade, $\neg(A \wedge \neg A)$ é um teorema de $L F I 1$. Assim, um par de fórmulas contraditórias, $A$ e $\neg A$, gera automaticamente um número infinito de outras contradições: $A \wedge \neg A$ e $\neg(A \wedge$ $\neg A),((A \wedge \neg A) \wedge \neg(A \wedge \neg A))$ e $\neg(A \wedge \neg A) \wedge \neg(A \wedge \neg A))$ etc.

${ }^{17}$ Para um discussão filosófica mais detalhada sobre a relação entre a lógica clássica e $L F I 1$, ver (Antunes 2019b).
} 
dicados que correspondem a essas propriedades satisfaçam o seguinte esquema:

(19) $\forall x_{1} \ldots \forall x_{n} \circ \mathbf{P}\left(x_{1}, \ldots, x_{n}\right)$,

onde $\mathbf{P}$ é um predicado arbitrário de $n$ lugares. Como consequência do fato sobre LFI1 mencionado acima, teríamos então que qualquer proposição envolvendo as propriedades em questão - e, portanto, que trate exclusivamente de objetos ontologicamente independentes - resultaria em trivialidade se contraditória. Contudo, o fato de muitas propriedades, como a de ser famoso, aplicarem-se indistintamente a objetos ontologicamente dependentes e independentes mostra que essa proposta está longe de ser ideal.

A solução consiste em restringir a aplicação do operador o àquelas instâncias de $\mathbf{P}$ que são ontologicamente independentes, o que pode ser feito através do esquema:

$$
\text { (20) } \forall x_{1} \ldots \forall x_{n}\left(I\left(x_{1}\right) \wedge \ldots \wedge I\left(x_{n}\right) \rightarrow \circ \mathbf{P}\left(x_{1}, \ldots, x_{n}\right)\right),
$$

onde $I$ é um predicado não lógico especial que expressa à propriedade de ser ontologicamente independente.

Definindo o predicado de ser ontologicamente dependente, $D$, por meio de:

$$
\text { (21) } D(t):=\neg I(t)
$$

(onde $t$ é uma constante ou variável individual) e assumindo $\forall x \circ I(x)-o$ que evita que um mesmo objeto seja simultaneamente ontologicamente dependente e independente - podemos provar através de (13) que (20) é equivalente a:

(22) $\forall x_{1} \ldots \forall x_{n}\left(\mathbf{P}\left(x_{1}, \ldots, x_{n}\right) \wedge \neg \mathbf{P}\left(x_{1}, \ldots, x_{n}\right) \rightarrow D\left(x_{1}\right) \vee \ldots \vee D\left(x_{n}\right)\right)$. 
(22) expressa que se $\mathbf{P}$ tem instâncias contraditórias, então a contradição em questão diz respeito a ao menos um objeto ontologicamente dependente.

Não é difícil provar que em LFII (20) e (22) podem ser generalizados para quaisquer fórmulas da linguagem, desde que alguns cuidados envolvendo os quantificadores sejam observados. As versões generalizadas de (20) e (22) afirmam respectivamente que não há contradições não explosivas sobre objetos ontologicamente independentes e, equivalentemente, que quaisquer contradições não explosivas sempre dizem respeito a ao menos algum objeto ontologicamente dependente. Ambas são a contraparte formal de (12) em $L F I 1$, pois proíbem, através da regra $E X P^{\circ}$, quaisquer contradições envolvendo proposições sobre objetos ontologicamente independentes.

\section{O papel da lógica clássica}

Em virtude do comportamento do operador $\circ$ em LFII, que permite recuperar a lógica clássica para as proposições que ocorrem em seu escopo, (20) (ou equivalentemente (22)) implica que qualquer inferência classicamente válida é também válida em $L F I 1$, contanto que as todas proposições envolvidas tratem exclusivamente de objetos ontologicamente independentes. Por outro lado, inferências clássicas que não satisfazem essa condição podem eventualmente não serem válidas. Como consequência, ao adotar (20) (ou (22)), podemos acomodar a lógica clássica no interior de LFI1. Dessa perspectiva, o raciocínio clássico nada mais é do que um caso particular do paraconsistente no qual todas as entidades sobre as quais se está racionando existem independentemente de nós, de nossas práticas linguísticas e de nossos processos psicológicos. Esse fato ilustra bem o aspecto multilógico das LFIs e $L F U s$, que permite lidar com diferentes contextos argumentativos, que estão sujeitos a diferentes relações de consequência lógica, em um único sistema.

Mas, afinal, se não há nada que proíba descrições contraditórias de objetos ontologicamente dependentes, por que então estamos, em geral, inclinados a encarar contradições com extrema suspeição, independentemente da natureza dos objetos sobre os quais elas dizem respeito? A resposta a essa pergunta tem a ver com a maneira como interagimos com objetos ontologicamen- 
te dependentes, pois na maior parte das vezes eles são produzidos de modo a refletir as características de objetos ontologicamente independentes. Por exemplo, uma personagem de ficção é em geral criada para representar de modo mais ou menos fidedigno as características de uma pessoa (ou animal) real. Assim, é natural, embora não seja necessário, que a personagem em questão acabe não sendo descrita de maneira contraditória. Esse fato explica também por que estamos frequentemente inclinados a pensar que inferências clássicas valem irrestritamente, independentemente do domínio sobre os quais estamos raciocinando - pois, como acabamos de ver, todas as inferências clássicas são válidas sempre que as proposições envolvidas tratam exclusivamente de objetos ontologicamente independentes.

Em suma: via de regra, interagimos com objetos ontologicamente dependentes como se eles fossem ontologicamente independentes, o que explica por que na maior parte das vezes resistimos erroneamente à ideia de que eles possam ter propriedades contraditórias e por que tomamos, também erroneamente, as inferências da lógica clássica como sendo irrestritamente válidas.

\section{Considerações finais}

Vimos aqui duas abordagens não dialeteístas à paraconsistência. Na primeira, que propõe uma interpretação epistêmica das lógicas paraconsistentes, uma contradição envolvendo $A$ e $\neg A$ indica a presença de evidências conflitantes, porém não conclusivas, para $A$ e $\neg A$. A ocorrência de contradições em um dado contexto argumentativo não significa, portanto, que contradições sejam verdadeiras, como propõem os dialeteístas, uma vez que a noção de evidência é mais fraca do que a de verdade. Na segunda abordagem, que possui inspiração em posições nominalistas em filosofia da matemática, contradições são autorizadas contanto que elas tratem de objetos ontologicamente dependentes, que, grosso modo, são aqueles produzidos por nossos processos psicológicos e práticas linguísticas.

Em ambas as abordagens, não há um conflito real entre as lógicas clássica e paraconsistente. Enquanto na interpretação epistêmica as diferenças entre as duas lógicas se devem ao fato de elas tratarem de noções de 
consequência lógica distintas (viz., preservação de verdade vs. preservação de evidência), na interpretação nominalista a lógica clássica é vista como um caso particular da lógica paraconsistente no qual todos os objetos envolvidos existem independentemente de nós. Além disso, a interpretação epistêmica combinada com o operador de classicalidade o permite recuperar todas as inferências clássicas para as proposições conclusivas.

Contradições produzidas por objetos ontologicamente dependentes podem, em certo sentido, ser consideradas verdadeiras. Mas nesse caso, não se trata de uma noção de verdade estritamente realista. Desse modo, alguém poderia, por exemplo, dizer que as proposições "as personagens de Jules e Jim são a mesma" e "as personagens de Jules e Jim não são a mesma" são ambas verdadeiras, dado que "Jules e Jim são a mesma pessoa" e "Jules e Jim não são a mesma pessoa" são verdadeiras na pequena história contada por (Everett 2005). Poder-se-ia, então, distinguir um dialeteísmo forte, relacionado a contradições ontologicamente independentes, de um dialeteísmo fraco, devido a contradições ontologicamente dependentes. Nós, entretanto, preferimos considerar que a atribuição de verdade a proposições sobre objetos ficcionais (e também a proposições sobre objetos ontologicamente dependentes) são antes apenas um 'modo de falar', do que uma posição legitimamente dialeteísta.

Embora seja uma tese controversa, o dialeteísmo representa uma importante posição em filosofia da lógica. Entretanto, o dialeteísmo não é a única e, de acordo com os autores, nem a melhor maneira de compreender a ocorrência de contradições em determinados contextos argumentativos. Como vimos aqui, existem diferentes formas de interpretá-las sem assumir que o mundo ou a realidade tenha aspectos intrinsecamente contraditórios de modo tornar algumas contradições verdadeiras.

\section{Referências}

de Amo, S., W. Carnielli, João Marcos. 2002. "A Logical Framework for Integrating Inconsistent Information in Multiple Databases." In Foundations of Information and Knowledge Systems, editado por Thomas Eiter e Klaus-Dieter Schewe, 67-84. Springer. 
Antunes, H. 2018. "On Existence, Inconsistency, and Indispensability." Principia: An International Journal of Epistemology 22: 7-34.

- 2019a. "Contradictions for Free: Towards a Nominalistic Interpretation of Contradictory Theories." Tese de Doutorado, Universidade de Campinas. https://bit.ly/antunes phdthesis.

- 2019b. "Enthymematic Classical Recapture." The Logic Journal of IGPL. https://doi.org/0.1093/jigpal/jzy061.

Antunes, H., W. Carnielli, A. Kapsner, A. Rodrigues. Kripke-style modelsfor logics of evidence and truth. Axioms, 9:1-16, 2020.

Aristotle. 1941. “Translation of Aristotle's Metaphysics.” In The Basic Works of Aristotle, editado por R. McKeon, traduzido por W. D. Ross. New York: Random House.

Azzouni, J. 2004. Deflating Existential Consequence: A Case for Nominalism. Oxford University Press.

- 2015. "Mathematical Fictions." In Fiction and Art: Explorations in Contemporary Theory, editado por Anantha Ch. Sukla, 63-77. Bloomsbury.

Batens, D. 2002. "In Defence of a Programme for Handling Inconsistencies." In Inconsistency in Science. Springer.

Belnap, N. D. 1977a. “A Useful Four-Valued Logic.” In Modern Uses of Multiple Valued Logics, editado por G. Epstein e J. M. Dunn. D. Reidel, Dordrecht.

1977b. "How a Computer Should Think." In Contemporary Aspects of Philosophy. Oriel Press.

Carnielli, W., M. E. Coniglio. 2016. Paraconsistent Logic: Consistency, Contradiction and Negation. Vol. 40 of Logic, Epistemology, and the Unity of Science series. Springer.

Carnielli, W., M. E. Coniglio, João Marcos. 2007. "Logics of Formal Inconsistency." In Handbook of Philosophical Logic (2nd. Edition), editado por D. M. Gabbay e F. Guenthner, 14:1-93. Springer.

Carnielli, W., M. E. Coniglio, A. Rodrigues. 2019. "Recovery Operators, Paraconsistency and Duality." Logic Journal of the IGPL. https://doi.org/ 10.1093/jigpal/jzy054.

Carnielli, W., João Marcos, S. de Amo. 2000. "Formal Inconsistency and Evolutionary Databases.” Logic and Logical Philosophy 8: 115-52. 
Carnielli, W., A. Rodrigues. 2016. "On the Philosophy and Mathematics of the Logics of Formal Inconsistency." In New Directions in Paraconsistent Logic, editado por J.-Y. Beziau, M. Chakraborty, e S. Dutta, 57-88. Springer.

- 2017. "An Epistemic Approach to Paraconsistency: A Logic of Evidence and Truth." Synthese 196: 3789-3813. https://doi.org/10.1007/s11229017-1621-7.

- 2020. "On Epistemic and Ontological Interpretations of Intuitionistic and Paraconsistent Paradigms." Logic Journal of the IGPL. https://doi.org/ 10.1093/jigpal/jzz041.

Chateaubriand, O. 2001. Logical Forms Vol. 1. Campinas: UNICAMP-CLE.

Colyvan, M. 2008. "The Ontological Commitments of Inconsistent Theories." Philosophical Studies 141: 115-23.

da Costa, N. C. A. 1963. "Sistemas Formais Inconsistentes (Inconsistent Formal Systems, in Portuguese)." Tese de Habilitação. Universidade Federal do Paraná, Curitiba, Brazil.

1974. "On the Theory of Inconsistent Formal Systems." Notre Dame Journal of Formal Logic XV (4): 497-510.

da Costa, N. C. A., e S. French. 2003. Science and Partial Truth: A Unitary Approach to Models and Scientific Reasoning. Oxford: Oxford University Press.

da Costa, N. C. A., D. Krause. 2014. "Physics, Inconsistency, and QuasiTruth." Synthese 191 (13): 3041-55.

Costa, N. C. A. da, D. Krause e O. Bueno. 2007. "Paraconsistent Logics and Paraconsistency." In Philosophy of Logic - Handbook of the Philosophy of Science Vol. 5, editado por D. Jacquette et al. Elsevier.

Dunn, J. M. 1976. "Intuitive Semantics for First-Degree Entailments and 'Coupled Trees'.” Philosophical Studies 29: 149-68.

Everett, A. 2005. "Against Fictional Realism.” The Journal of Philosophy 102: 624-49.

Frade, L., A. Rodrigues. 2020. "Some Remarks on Logical Realism and Logical Pluralism.” https://bit.ly/realism_pluralism.

Frápolli, M. 1996. "Review of Graham Priest 'Beyond the Limits of Thought'." The Review of Modern Logic, no. 6: 437-39. 
Frege, G. 1879. "Begriffsschrift, a Formula Language, Modeled Upon That of Arithmetic, for Pure Thought." In From Frege to gödel, editado por J. van Heijnoort, 1-82. Lincoln: toExcel Press (1999).

Garfield, J. 2004. "To Pee and Not to Pee? Could That Be the Question? (Further Reflections of the Dog)." In The Law of Non-Contradiction: New Philosophical Essays, editado por G. Priest, J. C. Beall, e B. Armour-Garb. Oxford University Press.

Gottlieb, P. 2013. “Aristotle on Non-Contradiction.” The Stanford Encyclopedia of Philosophy.

Kiefer, N. M., J. M. Dunn. 2019. "Contradictory Information: Better Than Nothing? The Paradox of the Two Firefighters." In Graham Priest on Dialetheism and Paraconsistency, editado por C. Başkent e T. M. Ferguson. Springer.

Łukasiewicz, J. 1910. "On the Principle of Contradiction in Aristotle." The Review of Metaphysics Vol. 24, No. 3 (1971): 485-509.

Mares, E. 2004. "Semantic Dialetheism." In The Law of Non-Contradiction: New Philosophical Essays, editado por Beall Priest e Armour-Garb. Oxford University Press.

Meheus, J., ed. 2002. Inconsistency in Science. Dordrecht: Springer.

Nickles, T. 2002. "From Copernicus to Ptolemy: Inconsistency and Method." In Inconsistency in Science, editado por J. Meheus. Dordrecht: Springer.

Omori, H., K. Sano. 2014. “da Costa Meets Belnap and Nelson.” In Recent Trends in Philosophical Logic, editado por R. Ciuni, H. Wansing, e C. Willkommen. Springer.

Omori, H., T. Waragai. 2011. "Some Observations on the Systems Lfi1 and Lfi1*." In Proceedings of Dexa2011, editado por F. Morvan, A. M. Tjoa, and R. Wagner, 320-24. IEEE Computer Society.

Popper, K. 1963. Conjectures and Refutations. New York, Harper.

Priest, G. 1998. "What Is so Bad about Contradictions?" Journal of Philosophy 95: 410-26.

- 2006. In Contradiction: A Study of the Transconsistent (2nd Edition). Oxford University Press.

—. 2019. "Some Comments and Replies." In Graham Priest on Dialetheism and Paraconsistency. 
Priest, G., F. Berto, W. Zach. 2018. "Dialetheism." The Stanford Encyclopedia of Philosophy (Fall 2018 Edition).

Rodrigues, A. 2017. "Uma Breve História Das Contradi: De Aristóteles à Paraconsistência." Nuntius Antiquus 13: 241-62.

Rodrigues, A., J. Bueno-Soler, W. Carnielli. 2020. "Measuring Evidence: A Probabilistic Approach to an Extension of Belnap-Dunn Logic.” Synthese. https://doi.org/10.1007/s11229-020-02571-w.

Sano, K., H. Omori. 2013. "An Expansion of First-Order Dunn-Belnap Logic." Logic Journal of the IGPL 22: 458-81.

Steinberger, F. 2016. "Explosion and the Normativity of Logic." Mind 125: 385-419.

Szmuc, D. 2016. "Defining LFIs and LFUs in Extensions of Infectious Logics." Journal of Applied Non-Classical Logics 26 (4): 286-314.

Tarski, A. 1956. "The Concept of Truth in Formalized Languages (1936).” In Logic, Semantics, Metamathematics. Clarendon Press.

Tugendhat, E., U. Wolf. 1996. Propedêutica lógico-Semântica. Petrópolis: Editora Vozes.

Wansing, H., S. Odintsov. 2016. "On the Methodology of Paraconsistent Logic." In Logical Studies of Paraconsistent Reasoning in Science and Mathematics, editado por H. Andreas e P. Verdée. Springer. 